Research Article

\title{
Topological Descriptors on Some Families of Graphs
}

\author{
Iftikhar Ahmad $\mathbb{D}^{1}$, Maqbool Ahmad Chaudhry, ${ }^{1}$ Muhammad Hussain $\mathbb{D}^{2}$, \\ and Tariq Mahmood ${ }^{3}$ \\ ${ }^{1}$ Department of Mathematics, The University of Lahore, Lahore, Pakistan \\ ${ }^{2}$ Department of Mathematics, COMSATS University Islamabad, Lahore Campus, Lahore, Pakistan \\ ${ }^{3}$ Department of Mathematics, Government College Gujranwala, Gujranwala, Pakistan
}

Correspondence should be addressed to Iftikhar Ahmad; iftikharahmadcheema072@gmail.com

Received 1 August 2021; Revised 15 September 2021; Accepted 28 September 2021; Published 21 October 2021

Academic Editor: Muhammad Kamran Jamil

Copyright (c) 2021 Iftikhar Ahmad et al. This is an open access article distributed under the Creative Commons Attribution License, which permits unrestricted use, distribution, and reproduction in any medium, provided the original work is properly cited.

\begin{abstract}
In view of the successful applications of graph theory, relationships between the biological activity and chemical structure have been developed. One of the popular topics in graph theory is problems relating to topological indices. Degree-based topological indices, distance-based topological indices, and counting-related topological indices are various types of topological indices. Physiochemical properties such as boiling point and stability of chemical compounds are correlated by these topological indices. A topological index of a graph is a numerical quantity obtained from the graph mathematically. A cactus graph is a connected graph in which no edge lies in more than one cycle. In this study, we have derived certain degree-based topological indices for some families of graphs consisting of graph obtained by the rooted product of paths and cycles and two types of cactus graph (paracactus and orthocactus) with the help of the generalized Zagreb index.
\end{abstract}

\section{Introduction}

Let $G$ be a graph, $V(G)$ be vertices of $G$, and $E(G)$ be the edge of $G$; then, the total number of vertices in $G$ is called the order of $G$, and the total number of edges in $G$ is called the size of $G$; any edge having the same starting and ending vertex is called a loop. In a graph, if two or more edges have the same starting and ending vertex, then we call this multiple edge. A graph which does not contain a loop or multiple edge is called a simple graph, and a graph which contains a loop or multiple edge is called a multigraph. A graph $G$ is a planar graph if we draw it into the plane without any edge intersection. If it is not possible, then we call it a nonplanar graph. In a graph $G$, from one vertex to another vertex, we give orientation or directions to each edge; then, this graph is called a directed graph; if we start moving from one vertex and, after travelling different edges, we reach back that vertex, then it forms a cycle. If a graph has no cycle, then we call it a tree. Spanning tree is a subgraph which has the same vertex as the original graph. In this paper, we use undirected graphs. Graph theory was successfully employed through the translation of chemical structures into characteristic numerical descriptors by resorting to graph invariants. A graph invariant is any function on a graph that does not depend on labeling of its vertices. Such quantities are also called topological indices. Hundreds of different invariants have been employed to date in QSAR/QSPR studies. Among more useful of them appear two that are known under various name. Topological indices are numerical parameters of a graph which are invariant under graph isomorphism. Interest in the field of computational chemistry in topological indices has been on the rise for a considerable length of time. A graph can be recognized by a numerical number, a polynomial, an arrangement of numbers, and either a network or a matrix which represents the whole graph. A topological index is a numerical amount related to a graph, which describes the geography of the graph and is invariant under diagram automorphism [1-7]. 
There are some significant classes of topological indices, for example, distance-based topological indices, degree-based topological indices, and eccentricity-based and countingrelated polynomials and indices of the graph. Among these classes, degree-based topological indices have overwhelming significance and perform a pivotal role in the preparation of graph hypothesis, especially in science. If it is made more precise, a topological list Top $(G)$ of a graph is a number with the property that, for each graph $H$ isomorphic to $G$, $\operatorname{Top}(H)=\operatorname{Top}(G)$. The concept of topological indices came from Wiener when he was studying the boiling point of a member of the alkene family, called paraffins. He named this topological index the path number. When research in chemical graph theory progressed, the name Wiener index was given to the path number. Owing to its interesting theoretical properties and wide range of applications, the Wiener index is the most investigated molecular topological index in chemical graph theory [8,9]. The first and the second Zagreb indices were introduced by Gutman and Trinajestic [10]. H. Wiener gave the concept of the topological index, namely, as the Wiener index [11] and is defined as

$$
W(G)=\frac{1}{2} \sum_{p q \in E(G)} d(p, q) .
$$

The chemist Randić introduced a topological index under the name branching index:

$$
R_{\alpha}(G)=\sum_{p q \in E(G)}[d(p) d(q)]^{\alpha} .
$$

Vukicevic and Furtula et al. introduced one of the wellknown connectivity topological indices, namely, atom-bond connectivity $(\mathrm{ABC})$ index [12], defined as

$$
\operatorname{ABC}(G)=\sum_{p q \in E(G)} \sqrt{\frac{d(p) d(q)-2}{d(p) d(q)}} .
$$

Nikolić et al. [13] introduced Zagreb indices defined as

$$
\begin{aligned}
& M_{1}(G)=\sum_{p \in V(G)} \delta(p)^{2}, \\
& M_{2}(G)=\sum_{p, q \in V(G)} \delta(p) \delta(q) .
\end{aligned}
$$

Vukičević and Furtula [12] introduced another wellknown connectivity topological descriptor, namely, geometric-arithmetic (GA) index:

$$
\mathrm{GA}(G)=\sum_{p q \in E(G)} \frac{2 \sqrt{d(p) d(q)}}{d(p)+d(q)}
$$

Some more degree-based topological indices are discussed in [14-16]. Aslam et al. computed topological indices of line graphs of subdivision graphs of the $i$ th vertex rooted product graphs [17]. Ahmad et al. computed polynomials of degree-based indices for swapped networks modeled by the optical transpose interconnection [1]. Ahmad computed degree-based topological indices of the benzene ring in the p-type surface in the $2 \mathrm{D}$ network [3].

$$
\begin{aligned}
& M_{a}(G)=(8 m n+4 m+4 n) 2^{a}+(16 m n-4 m-4 n) 3^{a}, \\
& R_{a}(G)=(4 m+4 n) 2^{2 a}+(16 m n-6 m-6 n) 3^{2 a}+(16 m n) 6^{a} .
\end{aligned}
$$

Vukicevic and Furtula computed the topological index based on the ratios of geometrical and arithmetical means of the end vertex degree of edges. In this paper, they introduced a novel topological index based on the end vertex degree and its basic features. They named it as the geometric-arithmetic index [12].

Farahani computed Zagreb indices and Zagreb polynomials of polycyclic aromatic hydrocarbons. In this paper, he computed the first Zagreb index $Z_{g_{1}}(G)$, second Zagreb index $Z_{g_{2}}(G)$, and their polynomials $Z_{g_{1}}(G, x)$ and $Z_{g_{2}}(G, x)$ of the family of hydrocarbon structure polycyclic aromatic hydrocarbons [5]. Sarkar et al. computed the general Zagreb index of some carbon structures. They computed the general Zagreb index for three carbon allotropes theoretically [15]. Sarkar et al. computed the general Zagreb index of some dendrimer structures. Dendrimers are generally large, complex, and hyperbranched molecules synthesized by repeatable steps with nanometer-scale measurements. They computed the general Zagreb index of some regular dendrimers and hence obtained some vertex degree-based topological indices [16]. Mustapha Chellali computed bounds on the 2-domination number in cactus graphs. He proved that if $G$ is a nontrivial connected cactus graph with $K(G)$ even cycles, then $\gamma_{2}(G) \geq \gamma_{t}(G)-K(G)$, and if $G$ is a graph of order $n$ with at most one cycle, then $\gamma_{2}(G) \geq(n+l-s) / 2$ improving Fink and Jacobson's lower bound for trees with $l>s$, where $\gamma_{t}, l$, and $s$ are the total domination number. He also proved that if $T$ is a tree of order $n \geq 3$, then $\gamma_{2} \leq \beta(T)+s-1[8]$.

\section{Main Results}

We derive the topological indices of the rooted product graph $C_{n}\left(P_{m}\right)$ of cycle and path graphs. In this work, the mathematical property of the general Zagreb index or $(s, t)$-Zagreb index of some general ortho- and paracactus chains is studied, and hence, their special cases such as triangular chain cactus $T_{n}$, orthochain square cactus $O_{n}$, and parachain square cactus $Q_{n}$ are considered where $n$ denotes the length of the chain, and then we derive some explicit expressions of the same for other degree-based topological indices such as Zagreb indices, forgotten index, redefined Zagreb index, general first Zagreb index, general Randić index, and symmetric division index for particular values of $s$ and $t$ of the general Zagreb index.

\section{Topological Indices of the Rooted Product Graph $C_{n}\left(P_{m}\right)$ of the Cycle and Path Graphs}

Let $C_{n}$ and $P_{m}$ be the cycle and path graphs on $n$ and $m$ vertices, respectively. Taking $n$ copies of $P_{m}$ and joining each vertex of $C_{n}$ with one vertex of $P_{m}$, we get the rooted product 


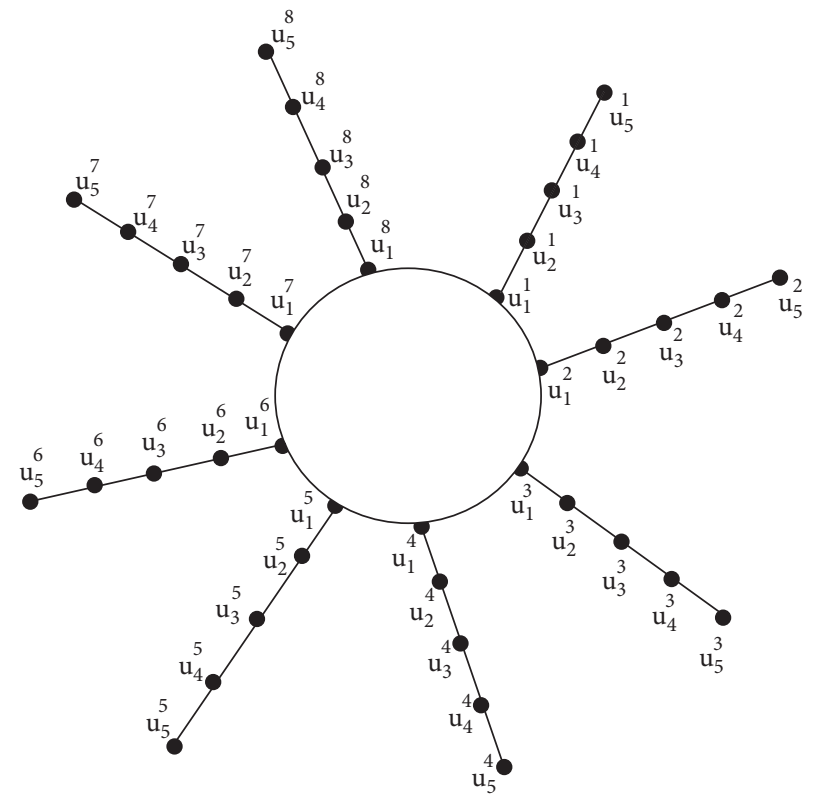

Figure 1: Example of the rooted product graph $C_{n}\left(P_{m}\right)$ for $n=8$ and $m=5$.

graph $C_{n}\left(P_{m}\right)$ of the cycle and path graphs. The edge set of $C_{n}\left(P_{m}\right)$ can be partitioned into the following subsets:

$$
\begin{aligned}
& E_{1}\left(C_{n}\left(P_{m}\right)\right)=\{e=p q ; d(p)=d(q)=3\}, \\
& E_{2}\left(C_{n}\left(P_{m}\right)\right)=\{e=p q ; d(p)=3, d(q)=2\}, \\
& E_{3}\left(C_{n}\left(P_{m}\right)\right)=\{e=p q ; d(p)=d(q)=2\}, \\
& E_{4}\left(C_{n}\left(P_{m}\right)\right)=\{e=p q ; d(p)=2, d(q)=1\},
\end{aligned}
$$

Proof

such that $\mid E_{1}\left(C_{n}\left(P_{m}\right)|=n, \quad| E_{2}\left(C_{n}\left(P_{m}\right)|=n, \quad| E_{3}\left(C_{n}\right.\right.\right.$ $\left(P_{m}\right) \mid=n(m-3)$, and $\mid E_{4}\left(C_{n}\left(P_{m}\right) \mid=n\right.$. In Figure 1 , the rooted product $C_{8}\left(P_{5}\right)$ is explained.

Theorem 1. If $C_{n}\left(P_{m}\right)$ is the rooted product graph of cycle and path graphs, then

(1) $M_{1}\left(C_{n}\left(P_{m}\right)\right)=M_{1,0}\left(C_{n}\left(P_{m}\right)\right)=4 n m+2 n$

(2) $M_{2}\left(C_{n}\left(P_{m}\right)\right)=1 / 2 M_{1,1}\left(C_{n}\left(P_{m}\right)\right)=4 n m+5 n$

(3) $F\left(C_{n}\left(P_{m}\right)\right)=M_{2,0}\left(C_{n}\left(P_{m}\right)\right)=8 n m+12 n$

(4) $R_{e} Z M\left(C_{n}\left(P_{m}\right)\right)=M_{2,1}\left(C_{n}\left(P_{m}\right)\right)=16 n m+42 n$

(5) $M^{\alpha}\left(C_{n}\left(P_{m}\right)\right)=M_{\alpha-1,0}\left(C_{n}\left(P_{m}\right)\right)=3^{\alpha} \times n+2^{\alpha} \times n \times$ $(m-2)+1^{\alpha} \times n$
(6) $R_{\alpha}\left(C_{n}\left(P_{m}\right)\right)=1 / 2 M_{\alpha, \alpha}\left(C_{n}\left(P_{m}\right)\right)=n(9)^{\alpha}+n(6)^{\alpha}+$ $n(m-3) n(4)^{\alpha}+n(2)^{\alpha}$

(7) $\operatorname{SDD} \quad\left(C_{n}\left(P_{m}\right)\right)=M_{1,-1}\left(C_{n}\left(P_{m}\right)\right)=2 n+13 n / 6+n$ $(m-3)(2)+5 / 2 n$

(1) We know that the corresponding $(s, t)$-Zagreb index of the first Zagreb index $M_{1}\left(C_{n}\left(P_{m}\right)\right)$ is $M_{1,0}\left(C_{n}\left(P_{m}\right)\right)$.

$$
\begin{aligned}
M_{1}\left(C_{n}\left(P_{m}\right)\right) & =M_{1,0}\left(C_{n}\left(P_{m}\right)\right)=? \\
M_{1}\left(C_{n}\left(P_{m}\right)\right) & =\sum_{p q \in E\left(C_{n}\left(P_{m}\right)\right)}\{d(p)+d(q)\} \\
& =n(3+3)+n(3+2)+n(m-3)(4)+n(3) \\
& =6 n+5 n+4 n m-12 n+3 n
\end{aligned}
$$

$M_{1}\left(C_{n}\left(P_{m}\right)\right)=4 n m+2 n$.

The general Zagreb index of $\left(C_{\backslash s n}\left(P_{m}\right)\right)$ is given by

$$
\begin{aligned}
M_{s, t}\left(C_{n}\left(P_{m}\right)\right)= & \sum_{p q \in E\left(C_{n}\left(P_{m}\right)\right)}\left\{d(p)^{s} d(q)^{t}+d(p)^{t} d(q)^{s}\right\} \\
= & n\left(3^{s} 3^{t}+3^{t} 3^{s}\right)+n\left(3^{s} 2^{t}+3^{t} 2^{s}\right)+n(m-3)\left(2^{s} 2^{t}+2^{t} 2^{s}\right)+n\left(2^{s} 1^{t}+2^{t} 1^{s}\right) . \\
M_{s, t}\left(C_{n}\left(P_{m}\right)\right)= & n\left(3^{s+t}+3^{s+t}\right)+n\left(3^{s} 2^{t}+3^{t} 2^{s}\right) \\
& +n(m-3)\left(2^{s+t}+2^{s+t}\right)+n\left(2^{s} 1^{t}+2^{t} 1^{s}\right) .
\end{aligned}
$$

Putting $s=1$ and $t=0$ in equation (1), we have 


$$
\begin{aligned}
M_{1,0}\left(C_{n}\left(P_{m}\right)\right)= & n(3+3)+n\left(3^{1} 2^{0}+3^{0} 2^{1}\right) \\
& +n(m-3)\left(2^{1}+2^{1}\right)+n\left(2^{1} 1^{0}+2^{0} 1^{1}\right) \\
= & n(6)+n(3+2)+n(m-3)(4)+n(3) \\
= & 6 n+5 n+4 n m-12 n+3 n \\
= & 4 n m+2 n .
\end{aligned}
$$

(2) We know that the corresponding $(s, t)$-Zagreb index of the second Zagreb index $M_{2}\left(C_{n}\left(P_{m}\right)\right)$ is $1 / 2 M_{1,1}\left(C_{n}\left(P_{m}\right)\right)$.

$$
\begin{aligned}
M_{2}\left(C_{n}\left(P_{m}\right)\right) & =\frac{1}{2} M_{1,1}\left(C_{n}\left(P_{m}\right)\right)=? \\
M_{2}\left(C_{n}\left(P_{m}\right)\right) & =\sum_{p q \in E\left(C_{n}\left(P_{m}\right)\right)} d(p) d(q) \\
M_{2}\left(C_{n}\left(P_{m}\right)\right) & =n(9)+n(6)+n(m-3)(4)+n(2) \\
& =9 n+6 n+4 n m-12 n+2 n \\
& =4 n m+5 n, \\
M_{1,1}\left(C_{n}\left(P_{m}\right)\right) & =n\left(3^{1} \times 3^{1}+3^{1} \times 3^{1}\right)+n\left(3^{1} \times 2^{1}+2^{1} \times 3^{1}\right)+n(m-3)\left(2^{1} \times 2^{1}\right. \\
& =n(9+9)+n(6+6)+n(m-3)(4+4)+n(2+2) \\
& =18 n+12 n+n(m-3)(8)+4 n \\
& =2[4 n m+5 n], \\
\frac{1}{2} M_{1,1}\left(C_{n}\left(P_{m}\right)\right) & =M_{2}\left(C_{n}\left(P_{m}\right)\right)=4 m n+5 n .
\end{aligned}
$$

(3) We know that the corresponding $(s, t)$-Zagreb index of the forgotten topological index $F\left(C_{n}\left(P_{m}\right)\right)$ is $M_{2,0}\left(C_{n}\left(P_{m}\right)\right)$.

$$
\begin{aligned}
F\left(C_{n}\left(P_{m}\right)\right)= & M_{2,0}\left(C_{n}\left(P_{m}\right)\right)=? \\
F\left(C_{n}\left(P_{m}\right)\right)= & \sum_{p q \in E\left(C_{n}\left(P_{m}\right)\right.}\left\{d(p)^{2}+d(q)^{2}\right\} \\
= & n\left(3^{2}+3^{2}\right)+n\left(3^{2}+2^{2}\right) \\
& +n(m-3)\left(2^{2}+2^{2}\right)+n\left(2^{2}+1^{2}\right) \\
= & 18 n+13 n+n(m-3)(8)+5 n \\
= & 8 m n+12 n .
\end{aligned}
$$

Putting $s=2$ and $t=0$ in equation (1),

$$
\begin{aligned}
M_{2,0}\left(C_{n}\left(P_{m}\right)\right)= & n\left(3^{2} \times 3^{0}+3^{0} \times 3^{2}\right)+n\left(3^{2} \times 2^{0}+3^{2} \times 2^{0}\right)+n(m-3)\left(2^{2} \times 2^{0}\right. \\
& \left.+2^{0} \times 2^{2}\right)+n\left(2^{2} \times 1^{0}+1^{2} \times 2^{0}\right) \\
= & n(9+19)+n(9+4)+n(m-3)(4+4)+n(4+1) \\
= & 18 n+13 n+8 m n-24 n+5 n \\
= & 8 m n+12 n, \\
M_{2,0}\left(C_{n}\left(P_{m}\right)\right)= & F\left(C_{n}\left(P_{m}\right)\right)=8 m n+12 n .
\end{aligned}
$$


(4) We know that the corresponding $(s, t)$-Zagreb index of the redefined Zagreb index $\operatorname{ReZM}\left(C_{n}\left(P_{m}\right)\right)$ is $M_{2,1}\left(C_{n}\left(P_{m}\right)\right)$.

$$
\begin{aligned}
\operatorname{Re} Z M\left(C_{n}\left(P_{m}\right)\right)= & M_{2,1}\left(C_{n}\left(P_{m}\right)\right)=? \\
\operatorname{Re} Z M\left(C_{n}\left(P_{m}\right)\right)= & \sum_{p q \in E\left(C_{n}\left(P_{m}\right)\right)} d(p) d(q)\{d(p)+d(q)\} \\
= & n(3)(3)(3+3)+n(3)(2)(3+2)+n(m-3)(2)(2)(2+2) \\
& +n(2)(1)(2+1) \\
= & n(9)(6)+n(6)(5)+n(m-3)(4)(4)+n(2)(3) \\
= & n(54)+n(30)+n(m-3)(16)+n(6) \\
& 54 n+30 n+16 m n-48 n+6 n .
\end{aligned}
$$

$\operatorname{Re} Z M\left(C_{n}\left(P_{m}\right)\right)=16 m n+42 n$.

Putting $s=2$ and $t=1$ in equation (1),

$$
\begin{aligned}
M_{2,1}\left(C_{n}\left(P_{m}\right)\right)= & n\left(3^{2} \times 3^{1}+3^{1} \times 3^{2}\right)+n\left(3^{2} \times 2^{1}+3^{1} \times 2^{2}\right)+n(m-3)\left(2^{2} \times 2^{1}\right. \\
& \left.+2^{1} \times 2^{2}\right)+n\left(2^{2} \times 1^{1}+1^{2} \times 2^{1}\right) \\
& n(9 \times 3+3 \times 9)+n(9 \times 2+3 \times 4)+n(m-3)(4 \times 2+2 \times 4) \\
& +n(4+2) \\
= & n(27+27)+n(18+12)+n(m-3)(8+8)+6 n, \\
= & 54 n+30 n+16 m n-48 n+6 n, \\
M_{2,1}\left(C_{n}\left(P_{m}\right)\right)= & 16 m n+42 n . \\
M_{2,1}\left(C_{n}\left(P_{m}\right)\right)= & \operatorname{Re} Z M\left(C_{n}\left(P_{m}\right)\right)=16 m n+42 n .
\end{aligned}
$$

(5) We know that the corresponding $(s, t)$-Zagreb index of the general Zagreb index $M^{\alpha}\left(C_{n}\left(P_{m}\right)\right)$ is $M_{\alpha-1,0}\left(C_{n}\left(P_{m}\right)\right)$.

$$
M^{\alpha}\left(C_{n}\left(P_{m}\right)\right)=M_{\alpha-1,0}\left(C_{n}\left(P_{m}\right)\right)=? .
$$

The general Zagreb index $M^{\alpha}\left(C_{n}\left(P_{m}\right)\right)$ is given by

$$
\begin{aligned}
& M^{\alpha}\left(C_{n}\left(P_{m}\right)\right)=\sum_{p \in V\left(C_{n}\left(P_{m}\right)\right)} d(p)^{\alpha} . \\
& M^{\alpha}\left(C_{n}\left(P_{m}\right)\right)=3^{\alpha} \times n+2^{\alpha} \times n \times(m-2)+1^{\alpha} \times n .
\end{aligned}
$$

Putting $s=\alpha-1$ and $t=0$ in equation (1), we get 


$$
\begin{aligned}
M_{\alpha-1,0}\left(C_{n}\left(P_{m}\right)\right)= & \left.n\left(3^{\alpha-1} \times 3^{0}+3^{0} \times 3^{\alpha-1}\right)+n\left(3^{\alpha-1}\right) \times 2^{0}+3^{0} \times 2^{\alpha-1}\right) \\
& +n(m-3)\left(2^{\alpha-1} \times 2^{0}+2^{0} \times 2^{\alpha-1}\right)+n\left(2^{\alpha-1} \times 1^{0}+1^{\alpha-1} \times 2^{0}\right) \\
= & n\left(2 \times 3^{\alpha-1}\right)+n\left(3^{\alpha-1}+2^{\alpha-1}\right)+n(m-3)\left(2 \times 2^{\alpha-1}\right)+n\left(2^{\alpha-1}\right. \\
& \left.+1^{\alpha-1} \times 2^{0}\right) \\
= & 2 n \times 3^{\alpha-1}+n 3^{\alpha-1}+n \times 2^{\alpha-1}+n m \times 2^{\alpha}-3 n \times 2^{\alpha} \\
& +n\left(2^{\alpha-1}+1^{\alpha-1} \times 1\right) \\
= & 3^{\alpha-1}[3 n]+2^{\alpha-1}[2 n]++n \times 1^{\alpha}+m n .2^{\alpha}-3 n \times 2^{\alpha} \\
= & 3^{\alpha} \times n+2^{\alpha} \times n+2^{\alpha} \times n[+m-3]+n \times 1^{\alpha} \\
= & 3^{\alpha} \times n+2^{\alpha} \times n \times(m-3)+1^{\alpha} \times n .
\end{aligned}
$$

(6) We know that the corresponding $(s, t)$-Zagreb index of the general Randić index $R_{\alpha}\left(C_{n}\left(P_{m}\right)\right)$ is $1 / 2 M_{\alpha, \alpha}\left(C_{n}\left(P_{m}\right)\right)$.

$$
R_{\alpha}\left(C_{n}\left(P_{m}\right)\right)=\frac{1}{2} M_{\alpha, \alpha}\left(C_{n}\left(P_{m}\right)\right)=? .
$$

The general Randić index $R_{\alpha}(G)$ is given by

$$
R_{\alpha}\left(C_{n}\left(P_{m}\right)\right)=\sum_{p q \in E\left(C_{n}\left(P_{m}\right)\right)}\{d(p) d(q)\}^{\alpha} \text {. }
$$

So,

$$
R_{\alpha}\left(C_{n}\left(P_{m}\right)\right)=n(9)^{\alpha}+n(6)^{\alpha}+n(2)^{\alpha}+n(m-3)(4)^{\alpha} .
$$

Using equation (1),

$$
\begin{aligned}
M_{s, t}\left(C_{n}\left(P_{m}\right)\right)= & n\left(3^{s+t}+3^{s+t}\right)+n\left(3^{s} 2^{t}+3^{t} 2^{s}\right) \\
& +n(m-3)\left(2^{s} 2^{t}+2^{t} 2^{s}\right) \\
& +n\left(2^{s} 1^{t}+2^{t} 1^{s}\right) .
\end{aligned}
$$

Putting $s=\alpha$ and $t=\alpha$ in equation (1), we have

$$
\begin{aligned}
M_{\alpha, \alpha}\left(C_{n}\left(P_{m}\right)\right)= & n\left(3^{2 \alpha}+3^{2 \alpha}\right)+n\left(3^{\alpha} \times 2^{\alpha}+3^{\alpha} \times 2^{\alpha}\right)+n(m-3)\left(2^{2 \alpha}+2^{2 \alpha}\right) \\
& +n\left(2^{\alpha} \times 1^{\alpha}+2^{\alpha} \times 1^{\alpha}\right) \\
= & n\left(2 \times 3^{2 \alpha}\right)+n\left(2 \times 2^{\alpha} 3^{\alpha}\right)+n(m-3)\left(2 \times 2^{2 \alpha}\right)+n\left(2 \times 2^{\alpha}\right) \\
= & 2\left\{n(9)^{\alpha}+n(6)^{\alpha}+n(m-3) n(4)^{\alpha}+n(2)^{\alpha}\right\}, \\
\frac{1}{2} M_{\alpha, \alpha}\left(C_{n}\left(P_{m}\right)\right)= & n(9)^{\alpha}+n(6)^{\alpha}+n(m-3) n(4)^{\alpha}+n(2)^{\alpha}, \\
\frac{1}{2} M_{\alpha, \alpha}\left(C_{n}\left(P_{m}\right)\right)= & R_{\alpha}\left(C_{n}\left(P_{m}\right)\right)=n(9)^{\alpha}+n(6)^{\alpha}+n(m-3) n(4)^{\alpha}+n(2)^{\alpha} .
\end{aligned}
$$

(7) We know that the corresponding $(s, t)$-Zagreb index of the symmetric division deg index $S D D\left(C_{n}\left(P_{m}\right)\right)$ is $M_{1,-1}\left(C_{n}\left(P_{m}\right)\right)$.

$$
\operatorname{SDD}\left(C_{n}\left(P_{m}\right)\right)=M_{1,-1}\left(C_{n}\left(P_{m}\right)\right)=? .
$$

Putting $s=1$ and $t=-1$ in equation (1), we have 


$$
\begin{aligned}
M_{1,-1}\left(C_{n}\left(P_{m}\right)\right)= & n\left(3^{1-1}+3^{1-1}\right)+n\left(3^{1} \times 2^{-1}+3^{-1} \times 2^{1}\right)+n(m-3)\left(2^{1-1}\right. \\
& \left.+2^{1-1}\right)+n\left(2^{1} \times 1^{-1}+1^{1} \times 2^{-1}\right) \\
= & n\left(3^{0}+3^{0}\right)+n\left(\frac{3}{2}+\frac{2}{3}\right)+n(m-3)\left(2^{0}+2^{0}\right)+n\left(2+\frac{1}{2}\right) \\
= & n(1+1)+n\left(\frac{9+4}{6}\right)+n(m-3)(1+1)+n\left(\frac{4+1}{2}\right), \\
M_{1,-1}\left(C_{n}\left(P_{m}\right)\right)= & 2 n+\frac{13 n}{6}+n(m-3)(2)+\frac{5 n}{2}, \\
S D D\left(C_{n}\left(P_{m}\right)\right)= & \quad \sum\left\{\frac{d(p)}{d(q)}+\frac{d(q)}{d(p)}\right\} \\
= & n\left(\frac{3}{3}+\frac{3}{3}\right)+n\left(\frac{3}{2}+\frac{2}{3}\right)+n(m-3)(2)+\frac{5 n}{2} \\
= & 2 n+\frac{13 n}{6}+n(m-3)(2)+\frac{5 n}{2}, \\
S D D\left(C_{n}\left(P_{m}\right)\right)= & M_{1,-1}\left(C_{n}\left(P_{m}\right)\right)=2 n+\frac{13 n}{6}+n(m-3)(2)+\frac{5 n}{2} . \\
S &
\end{aligned}
$$

\section{Topological Indices of Some General Cactus Chain Graphs}

In this section, we find topological indices of two general cactus chain graphs, namely, paracactus chain graph and orthocactus chain graph of cycles. We first consider the paracactus chain graph in which the cut vertices are not adjacent. The paracactus chain graph of cycles is denoted by $C_{m}^{n}$ where $m$ is the number of vertices of each cycle and $n$ is the length of the chain. The number of vertices of $C_{m}^{n}$ is $m n-n+1$, and the number of its edges is $m n$.

Theorem 2. Let $C_{m}^{n}$ be the paracactus chain graphs of cycles for $m \geq 3$ and $n \geq 2$; then,

$$
M_{(s, t)}\left(C_{m}^{n}\right)=4(n-1) 2^{s+t}\left(2^{s}+2^{t}\right)+(m n-4 n+4) 2^{s+t+1} \square
$$

Proof. The edge set of $C_{m}^{n}$ can be partitioned into the following subsets:

$$
\begin{aligned}
& E_{1}\left(C_{m}^{n}\right)=\left\{e=p q ; \mathrm{d} C_{m}^{n}(p)=\mathrm{d} C_{m}^{n}(q)=2\right\}, \\
& E_{2}\left(C_{m}^{n}\right)=\left\{e=p q ; \mathrm{d} C_{m}^{n}(p)=2, \mathrm{~d} C_{m}^{n}(q)=4\right\},
\end{aligned}
$$

such that $\left|E_{1}\left(C_{m}^{n}\right)\right|=2(m-2)+(m-4)(n-2)$ and $\left|E_{2}\left(C_{m}^{n}\right)\right|=4(n-1)$.

Then, for the general Zagreb index, we have

$$
\begin{aligned}
M_{s, t}\left(C_{m}^{n}\right) & =\sum_{\left.p q \in E\left(C_{m}^{n}\right)\right)}\left\{d(p)^{s} d(q)^{t}+d(p)^{t} d(q)^{s}\right\} \\
& =\sum_{p q \in E_{1}\left(C_{m}^{n}\right)}\left(2^{s} 2^{t}+2^{t} 2^{s}\right)+\sum_{p q \in E_{2}\left(C_{m}^{n}\right)}\left(2^{s} 4^{t}+2^{t} 4^{s}\right) \\
& =\left|E_{1}\left(C_{m}^{n}\right)\right|\left(2^{s} 2^{t}+2^{t} 2^{s}\right)+\left|E_{2}\left(C_{m}^{n}\right)\right|\left(2^{s} 4^{t}+2^{t} 4^{s}\right) \\
& =\{2(m-2)+(m-4)(n-2)\} \times 2^{s+t+1}+4(n-1) \times 2^{s+t}\left(2^{s}+2^{t}\right) \\
& =4(n-1) 2^{s+t}\left(2^{s}+2^{t}\right)+(m n-4 n+4) \times 2^{(s+t+1)} .
\end{aligned}
$$

Corollary 1. Let $C_{m}^{n}$ be the paracactus chain graph of cycles for $m \geq 3$ and $n \geq 2$; then,

(1) $M_{1}\left(C_{m}^{n}\right)=4 m n+8 n-8$

(2) $M_{2}\left(C_{m}^{n}\right)=4 m n+16 n-16$
(3) $X\left(C_{m}^{n}\right)=1 / 2\{m n-4 n(1-(\sqrt{2} / 3))+4(1-(\sqrt{2} / 3))\}$

(4) $A B C\left(C_{m}^{n}\right)=1 / \sqrt{2} m n-2 n(\sqrt{2}-\sqrt{3})+2(\sqrt{2}-\sqrt{3})$

(5) $S\left(C_{m}^{n}\right)=1 / 8 m n$

(6) $R_{(-1)}\left(C_{m}^{n}\right)=1 / 2(m n-2 n+2)$ 
(7) $R_{(-(1 / 2))}\left(C_{m}^{n}\right)=(1 / 2)\{m n-2 n(2-\sqrt{2})+2(2-\sqrt{2})\}$

Proof

$$
\begin{aligned}
M_{1}\left(C_{m}^{n}\right)= & \sum_{p q \in E\left(C_{m}^{n}\right)}\{d(p)+d(q)\} \\
= & 2(m-2)+(m-4)(n-2)(2+2)+4(n-1)(2+4) \\
= & (2 m-4+m n-2 m-4 n+8) 4+(4 n-4) 6 \\
M_{1}\left(C_{m}^{n}\right)= & 4 n m+8 n-8 \\
M_{2}\left(C_{m}^{n}\right)= & \sum_{p q \in E\left(C_{m}^{n}\right)} d(p) d(q) \\
= & (m n-4 n+4) 4+(4 n-4) 8 \\
= & 4 m n-16 n+16+32 n-32 M_{2}\left(C_{m}^{n}\right) \\
= & 4 n m+16 n-16
\end{aligned}
$$

(1) The sum-connectivity index of $C_{m}^{n}$ is

$$
\begin{aligned}
X\left(C_{m}^{n}\right)= & \sum_{p q \in E\left(C_{m}^{n}\right)}=\frac{1}{\sqrt{d(p)+d(q)}} \\
= & (m n-4 n+4) \frac{1}{\sqrt{4}}+(4 n-4) \frac{1}{\sqrt{6}} \\
= & \frac{1}{2}(m n-4 n+4)+\frac{1}{\sqrt{6}}(4 n-4) \\
= & \frac{1}{2} m n-2 n\left(1-\frac{\sqrt{2}}{\sqrt{3}}\right)+2\left(1-\frac{\sqrt{2}}{\sqrt{3}}\right) \\
& \frac{1}{2}\left\{m n-4 n\left(1-\frac{\sqrt{2}}{\sqrt{3}}\right)+4\left(1-\frac{\sqrt{2}}{\sqrt{3}}\right)\right\} .
\end{aligned}
$$

(2) The atom-bond connectivity index is given by

$$
\begin{aligned}
\operatorname{ABC}\left(C_{m}^{n}\right)= & \sum_{p q \in E\left(C_{m}^{n}\right)} \sqrt{\frac{d(p) \times d(q)-2}{d(p) \times d(q)}} \\
= & (m n-4 n+4) \sqrt{\frac{2 \times 2-2}{2 \times 2}} \\
& +(4 n-4) \sqrt{\frac{2 \times 4-2}{2 \times 4}} \\
= & (m n-4 n+4) \frac{1}{\sqrt{2}}+(4 n-4) \frac{\sqrt{3}}{2} \\
= & \frac{1}{\sqrt{2}} m n-2 n(\sqrt{2}-\sqrt{3})+(\sqrt{2}-\sqrt{3}) .
\end{aligned}
$$

(3) The Sanskruti index of $C_{m}^{n}$ is given by

$$
S\left(C_{m}^{n}\right)=\sum_{p q \in E\left(C_{m}^{n}\right)}\left\{\frac{d(p)+d(q)-2}{d(p) \times d(q)}\right\}^{3}
$$

$$
\begin{aligned}
= & (m n-4 n+4)\left(\frac{2+2-2}{2 \times 2}\right)^{3} \\
& +(4 n-4)\left(\frac{2+4-2}{2 \times 4}\right)^{3} \\
= & \frac{1}{8}\{m n-4 n+4+4 n-4\} \\
= & \frac{m n}{8} .
\end{aligned}
$$

$$
\begin{aligned}
R_{-1}\left(C_{m}^{n}\right) & =\sum_{p q \in E\left(C_{m}^{n}\right)} \frac{1}{d(p) \times d(q)} \\
& =(m n-4 n+4) \frac{1}{2 \times 2}+(4 n-4) \frac{1}{2 \times 4} \\
& =\frac{1}{4}(m n-4 n+4)+\frac{1}{8}(4 n-4) \\
& =\frac{1}{4}(m n-4 n+4+2 n-2) \\
& =\frac{1}{4}(m n-2 n+2) . \\
R_{-(1 / 2)}\left(C_{m}^{n}\right) & =\sum_{\left.p q \in E\left(C_{m}^{n}\right)\right)} \frac{1}{\sqrt{d(p) \times d(q)}} \\
& =(m n-4 n+4)\left(\frac{1}{\sqrt{4}}\right)+(4 n-4)\left(\frac{1}{\sqrt{8}}\right) \\
& \left.=\frac{1}{2}(m n-4 n+4)+(4 n-4) \frac{1}{2 \sqrt{2}}\right) \\
& =\frac{1}{2}\{m n-2 n(2-\sqrt{2})+2(2-\sqrt{2})\} . \\
& \\
& (m n-1
\end{aligned}
$$

If $m=4$, then we have the graph as shown in Figure 2 . If $m=6$, then we have the graph as shown in Figure 3 .

Putting $m=4$ and $m=6$ in equation (1), we get the desired results. Also, putting $m=4$ and $m=6$ in Corollary 1 , we get results for the above graph.

Now, consider the orthochain cactus graph of cycles where its cut vertices are adjacent. Let this type of cactus chain graph be denoted by $C O_{m}^{n}$ where $m$ is the length of each cycle and $n$ is the length of the chain. The number of vertices is $m n-n+1$, and the number of edges is $m n$. We calculate the Zagreb index of $\mathrm{CO}_{m}^{n}$.

Theorem 3. Let $\mathrm{CO}_{m}^{n}$ be the orthocactus chain graph of cycles for $m \geq 3$ and $n \geq 2$; then, 


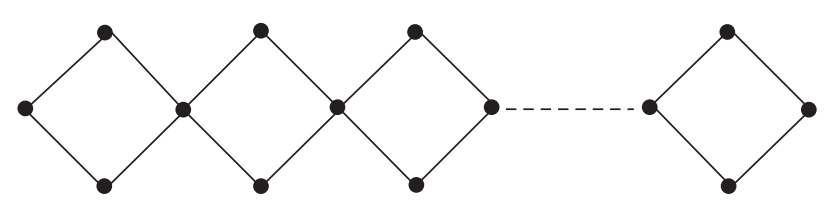

Figure 2: Example of the paracactus chain graph of cycles for $m=4$ and $n=n$.

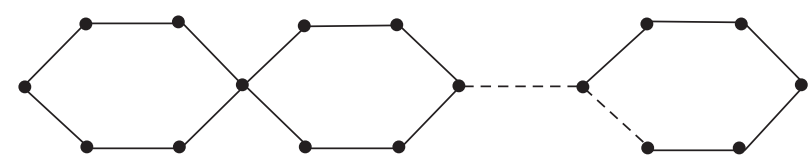

FiguRE 3: Example of the paracactus chain graph of cycles for $m=6$ and $n=n$.

$$
\begin{aligned}
M_{s, t}\left(C O_{m}^{n}\right)= & 2(n-2) 4^{(s+t)}+2 n \times 2^{(s+t)}\left(2^{s}+2^{t}\right) \\
& +2[2(m-2)+(m-3)(n-2)] 2^{(s+t)} .
\end{aligned}
$$$$
E_{1}\left(C O_{m}^{n}\right)=\{e=p q ; d(p)=d(q)=4\},
$$

Proof. The edge set of $\mathrm{CO}_{m}^{n}$ can be partitioned into the following subsets:

$$
\begin{aligned}
& E_{2}\left(C O_{m}^{n}\right)=\{e=p q ; d(p)=2, d(q)=4\}, \\
& E_{3}\left(C O_{m}^{n}\right)=\{e=p q ; d(p)=d(q)=2\},
\end{aligned}
$$

such that $\left|E_{1}\left(C O_{m}^{n}\right)\right|=(n-1), \quad\left|E_{2}\left(C O_{m}^{n}\right)\right|=2 n$, and $\left|E_{3}\left(C O_{m}^{n}\right)\right|=2(m-2)+(m-3)(n-2)$. The general Zagreb index is

$$
\begin{aligned}
M_{s, t}\left(C O_{m}^{n}\right) & =\sum_{p q \in E\left(C O_{m}^{n}\right)}\left\{d(p)^{s} d(q)^{t}+d(p)^{t} d(q)^{s}\right\} \\
& =\sum_{p q \in E\left(C O_{m}^{n}\right)}\left(4^{s} 4^{t}+4^{t} 4^{s}\right)+\sum_{p q \in E\left(C O_{m}^{n}\right)}\left(2^{s} 4^{t}+2^{t} 4^{s}\right)+\sum_{p q \in E\left(C O_{m}^{n}\right)}\left(2^{s} 2^{t}+2^{t} 2^{s}\right) \\
& =\left|E_{1}\left(C O_{m}^{n}\right)\right|\left(4^{s} 4^{t}+4^{t} 4^{s}\right)+\left|E_{2}\left(C O_{m}^{n}\right)\right|\left(2^{s} 4^{t}+2^{t} 4^{s}\right)+\left|E_{3}\left(C O_{m}^{n}\right)\right|\left(2^{s} 2^{t}+2^{t} 2^{s}\right) \\
& =(n-1)\left(4^{s} 4^{t}+4^{t} 4^{s}\right)+2 n\left(2^{s} 4^{t}+2^{t} 4^{s}\right)+2(m-2)+(m-3)(n-2)\left(2^{s} 2^{t}+2^{t} 2^{s}\right) \\
& =2(n-2) 4^{s+t}+2 n \times 2^{s+t}\left(2^{s+t}\right)+2\{(m-2)+(m-3)(n-2)\} 2^{s+t} \\
M_{s, t}\left(C O_{m}^{n}\right) & =2(n-2) 4^{s+t}+(m n-3 n+4) 2^{s+t+1}+2 n \times 2 s+t+1(2 s+2 t) .
\end{aligned}
$$

Corollary 2. Let $\mathrm{CO}_{m}^{n}$ be the orthocactus chain graph of cycles for $m \geq 3$ and $n \geq 2$. Then,

(1) $M_{1}\left(C O_{m}^{n}\right)=4 m n+8 n-8$

(2) $M_{2}\left(C O_{m}^{n}\right)=4 m n+20 n-24$

(3) $G A\left(\operatorname{COCO}_{m}^{n}\right)=m n-2 n(1-(2 \sqrt{2} / 3))$

(4) $X\left(C O_{m}^{n}\right)=1 / 2 m n+(n / 4 \sqrt{3})(\sqrt{6}+4 \sqrt{2}-6 \sqrt{3})+$ $(\sqrt{2}-1 / \sqrt{2})$
(5) $A B C\left(C O_{m}^{n}\right)=1 / \sqrt{2} m n+n / 4(4 \sqrt{3}+\sqrt{14}+6 \sqrt{2})+$ $(4-\sqrt{28} / 2 \sqrt{2})$

(6) $S\left(C O_{m}^{n}\right)=1 / 8 m n-37 / 512 n+37 / 256$

(7) $R_{-1}\left(C O_{m}^{n}\right)=1 / 16(4 m n-7 n+6)$

(8) $R_{-2}\left(C O_{m}^{n}\right)=1 / 2 m n+1 / 4 n(2 \sqrt{2}-5)+1 / 2$

Proof 


$$
\begin{aligned}
& M_{1}\left(C O_{m}^{n}\right)=\sum_{p q \in E\left(C O_{m}^{n}\right)}\{d(p)+d(q)\} \\
& =(n-2)(4+4)+2 n(2+4)+[2(m-2)+(m-3)(n-2)](2+2) \\
& =8 n-16+12 n+(2 m-4+m n-2 m-3 n+6) 4 \\
& =8 n-16+12 n+4 m n-12 n+8 \\
& =4 m n+8 n-8 . \\
& M_{2}\left(C O_{m}^{n}\right)=\sum_{p q \in E\left(\mathrm{CO}_{m}^{n}\right)}\{d(p) \times d(q)\} \\
& =(n-2)(4 \times 4)+2 n(2 \times 4)+(m n-3 n+2)(2 \times 2) \\
& =16 n-32+16 n+4 m n-12 n+8 \\
& =4 m n+20 n-24 \text {. } \\
& G A\left(C O_{m}^{n}\right)=\sum_{p q \in E\left(C O_{m}^{n}\right)} \frac{2 \sqrt{d(p) \times d(q)}}{d(p)+d(q)} \\
& =(n-2) \frac{2 \sqrt{4 \times 4}}{4+4}+2 n \frac{2 \sqrt{2 \times 4}}{2+4}+(m n-3 n+2) \times 2 \frac{\sqrt{2 \times 2}}{2+2} \\
& =(n-2) \frac{\sqrt{16}}{4}+2 n \frac{2 \sqrt{8}}{6}+(m n-3 n+2) \frac{\sqrt{4}}{2} \\
& =n-2+\frac{2}{3} n 2 \sqrt{2}+m n-3 n+2 \\
& =m n-2 n\left(1-\frac{2 \sqrt{2}}{3}\right) \text {. } \\
& X\left(C O_{m}^{n}\right)=\sum_{p q \in E\left(\mathrm{CO}_{m}^{n}\right)} \frac{1}{\sqrt{d(p)+d(q)}} \\
& =(n-2) \frac{1}{\sqrt{4+4}}+(2 n) \frac{1}{\sqrt{2+4}}+(m n-3 n+2) \frac{1}{\sqrt{2+2}} \\
& =(n-2) \frac{1}{\sqrt{8}}+(2 n) \frac{1}{\sqrt{6}}+(m n-3 n+2) \frac{1}{\sqrt{4}} \\
& \frac{1}{2} m n+\frac{n}{2 \sqrt{3}}(\sqrt{6}+4 \sqrt{2}-6 \sqrt{3})+\frac{\sqrt{2}-1}{\sqrt{2}} \text {. } \\
& \operatorname{ABC}\left(C_{m}^{n}\right)=\sum_{p q \in E\left(C O_{m}^{n}\right)} \sqrt{\frac{d(p) \times d(q)-2}{d(p) \times d(q)}} \\
& \left.=(n-2) \sqrt{\frac{4 \times 4-2}{4 \times 4}}\right)+(2 n) \sqrt{\frac{2 \times 4-2}{2 \times 4}}+(m n-3 n+2) \sqrt{\frac{2 \times 2-2}{2 \times 2}} \\
& =(n-2) \sqrt{\frac{14}{16}}+(2 n) \sqrt{\frac{3}{4}}+(m n-3 n+2) \sqrt{\frac{2}{4}} \\
& =\frac{m n}{\sqrt{2}}-\frac{n}{4}\left(\sqrt{14}+\frac{4}{\sqrt{3}}-\frac{12}{\sqrt{2}}\right)+\left(\frac{4-\sqrt{28}}{2 \sqrt{2}}\right) \\
& =\frac{m n}{\sqrt{2}}-\frac{n}{4}(4 \sqrt{3}+\sqrt{14}+6 \sqrt{2})+\left(\frac{4-\sqrt{28}}{2 \sqrt{2}}\right) \text {. }
\end{aligned}
$$




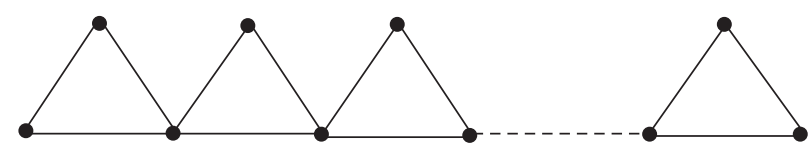

Figure 4: Example of the orthocactus chain graph of cycles for $m=3$ and $n=n$.

$$
\begin{aligned}
S\left(C O_{m}^{n}\right) & =\sum_{p q \in E\left(C O_{m}^{n}\right)}\left(\frac{d(p)+d(q)-2}{d(p) \times d(q)}\right)^{3} \\
& =(n-2)\left(\frac{4+4-2}{4 \times 4}\right)^{3}+2 n\left(\frac{2+4-2}{2 \times 4}\right)^{3}+(m n-3 n+2)\left(\frac{2+2-2}{2 \times 2}\right)^{3} \\
& =(n-2)\left(\frac{6}{16}\right)^{3}+2 n\left(\frac{4}{8}\right)^{3}+(m n-3 n+2)\left(\frac{2}{4}\right)^{3} \\
& =\frac{1}{8} m n-\frac{37}{512} n+\frac{37}{256} . \\
R_{-1}\left(C O_{m}^{n}\right) & =\sum_{p q \in E\left(C O_{m}^{n}\right)} \frac{1}{d(p) \times d(q)} \\
& =(n-2) \frac{1}{16}+\frac{2 n}{8}+(m n-3 n+2) \frac{1}{4} \\
& =\frac{m n}{4}+\frac{n}{16}+\frac{2 n}{8}-\frac{3 n}{4}+\frac{2}{4}-\frac{2}{16} \\
& =\frac{1}{16}(4 m n-7 n+6) . \\
R_{-2}\left(C O_{m}^{n}\right)= & \frac{m n}{2}+\frac{1}{4} n(2 \sqrt{2}-5)+\frac{1}{2} .
\end{aligned}
$$

Corollary 3. Let $T_{n}$ be the triangular cactus chain for $n \geq 2$; then,

$$
M_{s, t}\left(T_{n}\right)=2^{s+t+2}+n \times 2^{s+t+1}+2 \times(n-2) 4^{s+t} .
$$

Proof. Putting $m=3$ in equation (1), we get the desired result.

We have an orthocactus graph for $m=3$ and $n=n$ as shown in Figure 4.

Corollary 4. Let $T_{n}$ be the triangular cactus chain for $n \geq 2$; then,

(1) $M_{1}\left(T_{n}\right)=20 n-8$

(2) $M_{2}\left(T_{n}\right)=32 n-24$

(3) $G\left(T_{n}\right)=3 n-2 n(2 \sqrt{2} / 3)$

(4) $X\left(T_{n}\right)=3 / 2 n+n / 4 \sqrt{3} \quad(\sqrt{6}+4 \sqrt{2}-6 \sqrt{3})+$ $((\sqrt{2}-1) / \sqrt{2})$

(5) $A B C \quad\left(T_{n}\right)=3 / \sqrt{2} n+n / 4 \quad(4 / \sqrt{3}+\sqrt{14}+6 \sqrt{2})+$ $(4-\sqrt{28} / 2 \sqrt{2})$

(6) $S\left(T_{n}\right)=3 / 8 n-37 / 512 n+37 / 256$

(7) $R_{-1}\left(T_{n}\right)=12 / 16 n-7 / 16 n+6 / 16$

(8) $\left(R_{-2}\right)=3 / 2 n+1 / 4 n(2 \sqrt{2}-5)$
Proof. Putting $m=3$ in the results of Corollary 2, we get the desired results.

\section{Conclusion}

The topological indices such as the first Zagreb index, second Zagreb index, forgotten index, redefined Zagreb index, and general Randić index have been computed in this paper and have been compared with their corresponding $(s, t)$-Zagreb indices for the graph $C_{n}\left(P_{m}\right)$. In this study, some closed expressions of the general Zagreb index of some cactus chain graphs have also been obtained, leading to some other important degree-based topological indices for some particular values of $s$ and $t$. Results given by these indices can very much be correlated with molecular structures so as to understand their physical and chemical properties. The general Zagreb index of some other graph structures can be computed for further studies.

\section{Data Availability}

No data were used to support this study.

\section{Disclosure}

This paper has not been published elsewhere, and it will not be submitted anywhere else for publication. 


\section{Conflicts of Interest}

The authors declare that they have no conflicts of interest.

\section{Authors' Contributions}

All the authors contributed equally to this work.

\section{References}

[1] A. Ahmad, R. Hasni, K. Elahi, and M. A. Asim, "Polynomials of degree-based indices for swapped networks modelled by optical transpose interconnection system," IEEE Access, vol. 8, pp. 214293-214299, 2020.

[2] N. Ali, A. Koam, A. Ahmad, and M. F. Nadeem, "Comparative study of valency-based topological descriptor for hexagon star network," Computer Systems Science and Engineering, vol. 36, no. 2, pp. 293-306, 2021.

[3] A. Ahmad, "On the degree based topological indices of benzene ring embedded in the P-type-surface in 2D network," Hacettepe Journal of Mathematics and Statistic, vol. 47, no. 1, pp. 9-18, 2018.

[4] J. Cao, U. Ali, M. Javaid, and C. Huang, "Zagreb connection indices of molecular graphs based on operations," Complexity, vol. 2020, Article ID 7385682, 15 pages, 2020.

[5] M. .R. Farahani, "Zagreb indices and Zagreb polynomials of polycyclic aromatic hydro-carbons PAHs," Journal of Chemica Acta, vol. 2, pp. 70-72, 2013.

[6] M. Javaid and J. Cao, "Computing topological indices of probabilistic neural networks," Neural Computing and Applications, vol. 30, no. 12, pp. 3869-3876, 2018.

[7] H. Wang, M. Javaid, S. Akram, M. Jamal, and S. Wang, "Least eigenvalue of the connected graphs whose complements are cacti," Open Mathematics, vol. 17, no. 1, pp. 1319-1331, 2019.

[8] M. Chellali, "Bounds on the 2-dimensional number in cactus graph," Opuscula Mathematica, vol. 2, pp. 5-12, 2006.

[9] H. Wiener, "Structural determination of paraffin boiling points," Journal of the American Chemical Society, vol. 69, pp. 17-20, 1947.

[10] I. Gutman and N. Trinajestić, "Graph theory and molecular orbitals total p-electron energy of alternant hydrocarbons," Chemical Physics Letters, vol. 17, pp. 535-538, 1972.

[11] E. Estrada, L. Torres, L. Rodriguez, and I. Gutman, "An atombond connectivity index, modeling the enthalpy of formation of alkanes," Indian Journal of Chemistry, vol. 37A, pp. 849855, 1998.

[12] D. Vukicevic and B. Furtula, "Topological index based on the ratios of geometrical and arithmetic means of end-vertex degree of edges," Journal of Mathematical Chemistry, vol. 46, pp. 1369-1376, 2009.

[13] S. Nikolić, G. Kovačević, A. Miličević, and N. Trinajstić, "The Zagreb indices 30 years after," Croatica Chemica Acta, vol. 76, pp. 113-124, 2003.

[14] P. S. Ranjini, V. Lokesha, and A. Usha, "Relation between phenylene and hexagonal squeeze using harmonic index," International Journal of Graph Theory, vol. 1, pp. 116-121, 2013.

[15] P. Sarkar, N. De, and A. Pal, "The generalized Zagreb index of some carbon structures," Acta Chemica Iasi, vol. 26, no. 1, pp. 91-104, 2018.

[16] P. Sarkar, N. De, and A. Pal, "Generalized Zagreb index of some dendrimer structures," Universal Journal of Mathematics and Applications, vol. 1, no. 3, pp. 160-165, 2018.
[17] A. Aslam, M. Faisal Nadeem, Z. Zahid, S. Zafar, and W. Gao, "Computing certain topological indices of the line graphs of subdivision graphs of some rooted product graphs," Mathematics, vol. 7, p. 393, 2019. 